International Journal of Pure and Applied Mathematics

Volume 98 No. 1 2015, 129-143

ISSN: 1311-8080 (printed version); ISSN: 1314-3395 (on-line version)

url: http://www.ijpam.eu

doi: http://dx.doi.org/10.12732/ijpam.v98i1.11

ijpam.eu

\title{
CARATHEODORY DIFFERENTIAL EQUATIONS ON CONES
}

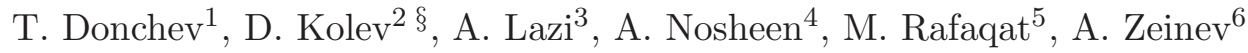 \\ ${ }^{1,3}$ Department of Mathematics \\ "Al. I. Cuza" University \\ Iaşi 700506, ROMANIA \\ ${ }^{2,6}$ Department of Mathematics \\ University of Chemical Technology and Metallurgy (UCTM) \\ 8 "St. Kl. Ohridski", Blvd., 1756 Sofia, BULGARIA \\ ${ }^{4,5}$ Abdus Salam School of Mathematical Sciences \\ 68-B, New Muslim Town, Lahore, PAKISTAN
}

\begin{abstract}
In this paper we prove that almost all, in Baire sense, differential equations with Scorza Dragoni right-hand side, defined on closed convex cone of a Banach space, have unique solution. This solution depends continuously on the right-hand side and on the initial condition. The results are applied to fuzzy differential equations and to differential inclusions.
\end{abstract}

AMS Subject Classification: $34 \mathrm{~A} 07,34 \mathrm{G} 20$

Key Words: differential equations, cone, Banach space, genericity, upper Lebesgue integral

\section{Introduction}

In this paper we study the following differential equation:

$$
\dot{x}(t)=f(t, x(t)), x(0)=x_{0}, t \in I=[0, T] .
$$

Here $f$ maps $I \times K$ into $K$, where $K$ is (arbitrary) nonempty closed convex cone in a nonseparable Banach space $X$.

Received: September 11, 2014

(C) 2015 Academic Publications, Ltd.

$\S$ Correspondence author url: www.acadpubl.eu 
It is proved in [10] that for almost all in Baire sense jointly continuous functions $f: I \times X \rightarrow X$ the problem (1.1) has unique noncontinuable solution, which depends continuously on $x_{0}$ and on $f$. The existence and uniqueness of solutions are proved for almost all Caratheodory functions $f$, when $X$ is a separable Hilbert space in [3], and in [4] when $X$ is a separable Banach space. The result of [10] is extended to the case of functional differential equations in [11]. In [16], using the theorem of Forth, the authors proved that for almost all jointly continuous with convex compact valued right-hand sides, the solution set of the corresponding differential inclusion depends continuously on the initial condition.

The extension of these results to the case of Caratheodory right hand sides in arbitrary (nonseparable) Banach space has not been investigated so far. This question is interesting itself, moreover, the extensively studied fuzzy differential equations as well as multivalued differential equations with Hukuhara derivative can be represented as abstract differential equations on closed convex cone in a (nonseparable) Banach space.

The function $p: I \rightarrow K$ is said to be strongly measurable if it is a limit of step functions for a.a. $t \in I$. Due to Lusin's property $p(\cdot)$ is strongly measurable if and only if for every $\varepsilon>0$ there exists an open set $I_{\varepsilon}$ with measure meas $\left(I_{\varepsilon}\right)<\varepsilon$ such that $p$ is continuous on $I \backslash I_{\varepsilon}$.

Recall that the function $f: I \times K \rightarrow K$ is said to be continuous at $(t, x)$ if for every $\varepsilon>0$ there exists $\delta>0$ such that $|f(t, x)-f(s, y)|<\varepsilon$ when $|t-s|+|x-y|<\delta$. The function $f(\cdot, \cdot)$ is said to be Caratheodory if $f(\cdot, x)$ is strongly measurable for every $x \in K$ and $f(t, \cdot)$ is continuous for a.a. $t \in I$. Further, $f(\cdot, \cdot)$ is said to be almost continuous (Scorza Dragoni) when for every $\varepsilon>0$ there exists an open set $I_{\varepsilon}$ with Lebesgue measure meas $\left(I_{\varepsilon}\right)<\varepsilon$ such that $f(\cdot, \cdot)$ is continuous on $\left(I \backslash I_{\varepsilon}\right) \times K$.

Let $B \subset K$. Denote by $\operatorname{Car}(B)$ and $C_{S D}(B)$ the set of all Caratheodory, respectively Scorza Dragoni functions from $I \times B$ into $K$, which are integrally bounded on bounded sets (i.e. for any bounded subset $U \subset B$ there exists $\lambda \in L_{1}(I)$ such that $|f(t, x)| \leq \lambda(t)$ for a.a. $t \in I$ and all $\left.x \in U\right)$.

Notice that $K$ is cone and $x-y \notin K$ in general for $x, y \in K$, however, $x-y \in X$ and it is not a confusion. We will write $x \ominus y$, to point out that the difference belongs to $K$.

\section{The goals of the paper are:}

1) Defining of an appropriate metric such that $\operatorname{Car}(K)$ and $C_{S D}(K)$ become complete metric spaces.

2) One of our (in some sense surprising) results states that either $C_{S D}(K) \equiv$ $\operatorname{Car}(K)$, or $C_{S D}(K)$ is closed nowhere dense subset of $\operatorname{Car}(K)$, i.e. of the first 
Baire category.

3) We prove that the existence and uniqueness of the solution is a residual property w.r.t. the so defined metric in the space of Scorza Dragoni functions. This result is then applied to the case of set valued differential equations and differential inclusions and also to the case of fuzzy systems.

Next we give some definitions, notations and recall some needed results.

Suppose $\mathbb{B}$ is the unit ball in $K$, i.e. $x \in K$ with $|x| \leq 1$. The ball with center at $x_{0}$ and radius $M$ is denoted by $\mathbb{B}_{M}=: x_{0}+M \mathbb{B}$.

Let $\psi: I \rightarrow \mathbb{R}_{+}$be integrally bounded function. Then the upper Lebesgue integral is:

$$
\bar{\int}_{I} \psi(t) d t=\inf \left\{\int_{I} \alpha(t) d t, \alpha(t) \geq \psi(t), \alpha(\cdot) \in L_{1}\left(I, \mathbb{R}_{+}\right)\right\}
$$

Proposition 1.1. For every positive integrally bounded function $\psi(\cdot)$ there exists $\alpha(\cdot) \in L_{1}\left(I, \mathbb{R}_{+}\right)$such that $\alpha(s) \geq \psi(s)$ for a.a. $s \in I$ and $\int_{0}^{t} \alpha(s) d s=\overline{\int_{0}^{t}} \psi(s) d s$, for every $t \in I$.

Proof. Let $\varepsilon_{n}=\frac{1}{2^{n}}$, then there exists $\alpha_{n}(\cdot) \in L_{1}\left(I, \mathbb{R}_{+}\right)$such that $\alpha_{n}(t) \geq$ $\psi(t)$ for a.a. $t \in I$ and $\int_{I} \alpha_{n}(t) d t \leq \bar{\int}_{I} \psi(t) d t+\varepsilon_{n}$, for any $n \geq 1$.

Define $\alpha(t)=\inf _{n \geq 1}\left\{\alpha_{n}(t)\right\}$. Then $\alpha(t) \geq \psi(t)$ and $\int_{I} \alpha(t) d t \leq \bar{\int}_{I} \psi(t) d t+$ $\varepsilon_{n}$, for any $n \geq 1$, hence $\int_{I} \alpha(t) d t=\bar{\int}_{I} \psi(t) d t$. Consequently, $\int_{0}^{t} \alpha(s) d s=$ $\overline{\int_{0}^{t}} \psi(s) d s$, for every $t \in I$.

The metric in $\operatorname{Car}\left(\mathbb{B}_{M}\right)$ is defined by the upper Lebesgue integral, i.e. for $f, g \in \operatorname{Car}\left(\mathbb{B}_{M}\right)$,

$$
\rho_{M}(f, g)=\overline{\int_{I}} \sup _{x \in \mathbb{B}_{M}}|f(t, x)-g(t, x)| d t
$$

Notice that $\left.t \rightarrow \sup _{x \in \mathbb{B}_{M}} \mid f(t, x)-g(t, x)\right) \mid$ is not measurable in general. However, it is integrally bounded and hence the upper Lebesgue integral exists. 
The space $\operatorname{Car}(K)$ is provided with the metric:

$$
\rho(f, g)=\sum_{M=1}^{\infty} \frac{\rho_{M}(f, g)}{2^{M}\left(1+\rho_{M}(f, g)\right)} .
$$

Notice that $f(\cdot, \cdot)$ is Scorza Dragoni $\left(f \in C_{S D}(K)\right)$ if and only if it is Scorza Dragoni on $I \times \mathbb{B}_{M}\left(f \in C_{S D}\left(\mathbb{B}_{M}\right)\right)$ for every natural $M$. Also, $\lim _{n \rightarrow \infty} \rho\left(f_{n}, f\right)=0$ if and only if $\lim _{n \rightarrow \infty} \rho_{M}\left(f_{n}, f\right)=0$ for every natural $M$.

\section{The Set of Scorza Dragoni Functions}

In this section we prove that the set of all Scorza Dragoni functions $C_{S D}(K)$ is a complement of the set of all continuous functions from $I \times K$ into $K$. Furthermore, we show that $C_{S D}(K)$ is in general closed nowhere dense subset of the space of Caratheodory functions $\operatorname{Car}(K)$.

Theorem 2.1. The class $C_{S D}(K)$ is a complete metric space w.r.t. $\rho$.

Proof. First, we show that $\rho$ is a distance, i.e. $C_{S D}(K)$ is a metric space.

Let $\rho(f, g)=0$, then $\sup _{x \in \mathbb{B}_{M}}|f(t, x)-g(t, x)|=0$ for a.a. $t \in I$ and every $M \in \mathbb{N}$, i.e. there exists a null set $N_{M}$ such that $f(t, x)=g(t, x)$, for every $t \in\left(I \backslash N_{M}\right)$ and every $x \in \mathbb{B}_{M}$. Let $N=\bigcup_{M=1}^{\infty} N_{M}$, then it is easy to see that $f(t, x)=g(t, x)$ for $(t, x) \in(I \backslash N) \times K$. Since $\rho(f, g)=\rho(g, f)$, it remains to prove the triangle inequality. Fix $M \in \mathbb{N}$ for our purpose and then should prove that the triangle inequality holds for $\rho_{M}(\cdot, \cdot)$. Let $f, g, h \in C_{S D}\left(\mathbb{B}_{M}\right)$. Clearly

$$
\sup _{x \in \mathbb{B}_{M}}|f(t, x)-g(t, x)| \leq \sup _{x \in \mathbb{B}_{M}}|f(t, x)-h(t, x)|+\sup _{x \in \mathbb{B}_{M}}|h(t, x)-g(t, x)| .
$$

Consequently, $\rho_{M}(f, g) \leq \rho_{M}(f, h)+\rho_{M}(h, g)$.

Now we will prove that $C_{S D}\left(\mathbb{B}_{M}\right)$ is complete and at the same time will actually show that it is completion of the space of the jointly continuous functions w.r.t. $\rho_{M}$.

First, we will prove that every Scorza Dragoni function is a limit of a sequence of continuous functions.

Let $f(\cdot, \cdot) \in C_{S D}\left(\mathbb{B}_{M}\right)$. Then having in mind the Proposition 1.1, conclude that there exists a real valued Lebesgue integrable function $\lambda(\cdot)$ such that $\sup _{x \in \mathbb{B}_{M}}|f(t, x)| \leq \lambda(t)$ for a.a. $t \in I$ and $\int_{0}^{t} \lambda(s) d s=\overline{\int_{0}^{t}} \sup _{x \in \mathbb{B}_{M}}|f(s, x)| d s$ for 
every $t \in I$. Furthermore, for every $\varepsilon>0$ there exists an open set $I_{\varepsilon} \subset I$ with Lebesgue measure meas $\left(I_{\varepsilon}\right)<\varepsilon$ such that $f(\cdot, \cdot)$ is continuous on $\left(I \backslash I_{\varepsilon}\right) \times \mathbb{B}_{M}$. Fix $\delta>0$ and choose $\varepsilon$ to be such that $\int_{J} \lambda(t) d t<\frac{\delta}{10}$ for every $J \subset I$ with $\operatorname{meas}(J)<\varepsilon$.

We can assume without loss of generality that $\lambda(\cdot)$ is continuous on $I \backslash I_{\varepsilon}$ and hence there exists $\mu>0$ such that $\lambda(t) \leq \mu$ for every $t \in\left(I \backslash I_{\varepsilon}\right)$.

It is known that $I_{\varepsilon}=\bigcup_{k=1}^{\infty} I_{k}$, where $I_{k}=\left(a_{k}, b_{k}\right)$ are open pairwise disjoint intervals. Then take $\tilde{a}_{k}>a_{k}$ and $\tilde{b}_{k}<b_{k}$, for every $k \geq 1$, such that $\sum_{k=1}^{\infty} \mid \tilde{a}_{k}-$ $a_{k}+b_{k}-\tilde{b}_{k} \mid<\frac{\delta}{10 \mu}$. There exists $m \geq 1$ such that $\sum_{k=m+1}^{\infty}\left(\tilde{b}_{k}-\tilde{a}_{k}\right)<\frac{\delta}{10 \mu}$.

Next for $\tilde{I}=\bigcup_{k=1}^{m}\left[\tilde{a}_{k}, \tilde{b}_{k}\right]$ the known Theorem of Dugundji (see [6]) implies that there exists a continuous function $\tilde{f}(\cdot, \cdot)$ on $I \times \mathbb{B}_{M}$ such that

$$
\tilde{f}(t, x)= \begin{cases}f(t, x) & (t, x) \in(I \backslash I \varepsilon) \times \mathbb{B}_{M}, \\ \hat{0} & (t, x) \in \tilde{I} \times \mathbb{B}_{M} .\end{cases}
$$

Consequently,

$$
\bar{\int}_{I} \sup _{x \in \mathbb{B}_{M}}|\tilde{f}(t, x)-f(t, x)| d t \leq \int_{\tilde{I}} \lambda(t) d t+2 \mu \sum_{k=1}^{m}\left(\tilde{a}_{k}-a_{k}+b_{k}-\tilde{b}_{k}\right)+\mu \sum_{k=m+1}^{\infty}\left(\tilde{b}_{k}-\tilde{a}_{k}\right) .
$$

Thus $\rho_{M}(\tilde{f}, f)<\frac{\delta}{10}+\frac{2 \mu \delta}{10 \mu}+\frac{\mu \delta}{10 \mu}<\delta$.

Second, we will prove that every Cauchy sequence $\left\{f_{m}(\cdot, \cdot)\right\}_{m=1}^{\infty}$ of continuous functions has a limit w.r.t. $\rho_{M}$, which belongs to $C_{S D}\left(\mathbb{B}_{M}\right)$.

Let $\varepsilon>0$, then there exists $N_{0}$ such that $\rho_{M}\left(f_{n}, f_{m}\right)<\varepsilon$ when $m, n \geq N_{0}$. We set $g_{0}(t, x)=f_{N_{0}}(t, x)$, and by induction take $\varepsilon_{k}=\frac{\varepsilon}{2^{k}}$. There exists $N_{k}$ such that $\rho_{M}\left(f_{n}, f_{m}\right)<\varepsilon_{k}$ for $n, m \geq N_{k}$. Denote $g_{k}(t, x)=f_{N_{k}}(t, x)$, thus $\sum_{m=k+1}^{\infty} \rho_{M}\left(g_{m}, g_{m+1}\right) \leq \varepsilon_{k}$.

Next fix $x \in \mathbb{B}_{M}$ and then since $\left\|g_{m}(\cdot, x)-g_{m+1}(\cdot, x)\right\|_{L_{1}} \leq \varepsilon_{m}$, one has that $\left\{g_{m}(\cdot, x)\right\}_{m=1}^{\infty}$ is a Cauchy sequence in $L_{1}\left(I, \mathbb{B}_{M}\right)$. Thus there exists $g_{x}(t)=$ 
$\lim _{k \rightarrow \infty} g_{k}(t, x)$ for a.a. $t \in I$. Define $f(t, x)=g_{x}(t)$ (recall that $x$ is fixed). Therefore

$$
\lim _{k \rightarrow \infty} \int_{I} \sup _{x \in \mathbb{B}_{M}}\left|f_{k}(t, x)-f(t, x)\right| d t=0 .
$$

Then, for any $k \geq 1$ there exists $\lambda_{k}(\cdot) \in L_{1}(I)$ such that $\sup _{x \in \mathbb{B}_{M}} \mid f_{k}(t, x)-$ $f(t, x) \mid \leq \lambda_{k}(t)$ and $\lim _{k \rightarrow \infty} \int_{I} \lambda_{k}(t) d t=0$. Passing to subsequences if necessary $\lambda_{k}(t) \rightarrow 0$ for a.a. $t \in I$ as $k \rightarrow \infty$. Thus by Egorov's theorem for every $\varepsilon>0$ there exists an open set $I_{\varepsilon} \subset I$ with meas $\left(I_{\varepsilon}\right)<\varepsilon$ such that $\lambda_{k}(t) \rightarrow 0$ uniformly on $\left(I \backslash I_{\varepsilon}\right)$ and hence $f_{k}(\cdot, \cdot)$ converges to $f(\cdot, \cdot)$ uniformly on $\left(I \backslash I_{\varepsilon}\right) \times \mathbb{B}_{M}$. Thus $f(\cdot, \cdot)$ is continuous on $\left(I \backslash I_{\varepsilon}\right) \times \mathbb{B}_{M}$, hence $f(\cdot, \cdot) \in C_{S D}\left(\mathbb{B}_{M}\right)$.

Now we will prove a similar result for $\operatorname{Car}\left(\mathbb{B}_{M}\right)$.

Theorem 2.2. The set $\operatorname{Car}\left(\mathbb{B}_{M}\right)$ is a complete metric space w.r.t. $\rho_{M}$.

Proof. Let $\left\{f_{k}(\cdot, \cdot)\right\}_{k=1}^{\infty}$ be a Cauchy sequence in $\operatorname{Car}\left(\mathbb{B}_{M}\right)$. Fix $\bar{x} \in \mathbb{B}_{M}$ and then conclude that $\left\{f_{k}(\cdot, \bar{x})\right\}_{k=1}^{\infty}$ is a Cauchy sequence in $L_{1}(I)$, because $\left|f_{k}(t, \bar{x})-f_{m}(t, \bar{x})\right| \leq \sup _{x \in \mathbb{B}_{M}}\left|f_{k}(t, x)-f_{m}(t, x)\right|$. Thus $f_{k}(\cdot, \bar{x}) \rightarrow y_{\bar{x}}(\cdot)$, which is $L_{1}$ function and hence strongly measurable.

Define $f(t, x)=y_{x}(t)$. As it was shown in the proof of Theorem 2.1, for every $\varepsilon>0$ there exists a compact set $I_{\varepsilon} \subset I$ with meas $\left(I \backslash I_{\varepsilon}\right)<\varepsilon$ such that $f_{k}(t, x) \rightarrow f(t, x)$ uniformly on $I_{\varepsilon} \times \mathbb{B}_{M}$. The latter implies that $f(t, \cdot)$ is continuous for every $t \in I_{\varepsilon}$. Since $\varepsilon>0$ is arbitrary, one has that $f(t, \cdot)$ is continuous for a.a. $t \in I$.

The next theorem is interesting itself and can be considered as the first main result of the paper.

Theorem 2.3. $C_{S D}\left(\mathbb{B}_{M}\right)$ is either a closed with empty interior subset of $\operatorname{Car}\left(\mathbb{B}_{M}\right)$ w.r.t. $\rho_{M}$ or $C_{S D}\left(\mathbb{B}_{M}\right) \equiv \operatorname{Car}\left(\mathbb{B}_{M}\right)$.

Proof. Since $C_{S D}\left(\mathbb{B}_{M}\right)$ is complete, then it is a closed subset of $\operatorname{Car}\left(\mathbb{B}_{M}\right)$. It is well known that $C_{S D}(K) \equiv \operatorname{Car}(K)$ when $K$ is separable, however, in general this is not the case when $K$ is not separable.

Let $\operatorname{Car}\left(\mathbb{B}_{M}\right) \backslash C_{S D}\left(\mathbb{B}_{M}\right) \neq \emptyset$.

Notice that the sum of Scorza Dragoni functions is also Scorza Dragoni . Let $f(\cdot, \cdot)$ be Scorza Dragoni and $g(\cdot, \cdot)$ be Caratheodory (not Scorza Dragoni). Consider the sequence $f_{k}(t, x)=\left(1-\frac{1}{k}\right) f(t, x)+\frac{1}{k} g(t, x)$. The func- 
tion $f_{k}(\cdot, \cdot)$ is Caratheodory, but not Scorza Dragoni for every $k$. Furthermore, $\lim _{k \rightarrow \infty} \rho_{M}\left(f_{k}, f\right)=0$ and hence the set $C_{S D}\left(\mathbb{B}_{M}\right)$ is with empty interior in $\operatorname{Car}\left(\mathbb{B}_{M}\right)$ w.r.t. $\rho_{M}$.

Notice that Theorem 2.3 implies that the set $C_{S D}\left(\mathbb{B}_{M}\right)$ is either of first Baire category on $\operatorname{Car}\left(\mathbb{B}_{M}\right)$ or the both sets coincide as in the case of separable $K$.

Corollary 2.4. The set of all locally Lipschitz functions on $I \times \mathbb{B}_{M}$ is dense in $C_{S D}\left(\mathbb{B}_{M}\right)$.

Proof. Actually, using the proof of Lemma 1 in [10] with obvious modifications where it is needed, one can show that for every continuous $f(\cdot, \cdot)$ and every $\varepsilon>0$ there exists a locally Lipschitz $g(\cdot, \cdot)$ such that $|f(t, x)-g(t, x)|<\varepsilon$, $\forall(t, x) \in I \times \mathbb{B}_{M}$, which implies that $\rho_{M}(f, g) \leq T \varepsilon$. However $C\left(I \times \mathbb{B}_{M}, K\right)$ is dense in $C_{S D}\left(\mathbb{B}_{M}\right)$. The proof is therefore complete.

\section{Existence and Uniqueness for Differential Equations on Cones}

In this section we will show that every differential equation on cone admits local $\varepsilon$-solution. Further we show that for almost every (in Baire sense) $f \in$ $C_{S D}\left(\mathbb{B}_{M}\right)$ the equation (1.1) admits an unique solution extendable to the boundary of $I \times \mathbb{B}_{M}$, for every natural $M$.

Denote by $\mathcal{M}$ the set of all $f(\cdot, \cdot) \in C_{S D}(K)$ such that (1.1) has a unique (noncontinuable) solution. Also, $\mathcal{M}_{M}$ is the set of $f(\cdot, \cdot) \in C_{S D}\left(\mathbb{B}_{M}\right)$ such that (1.1) has unique solution $y(\cdot)$ defined on $\left[0, T_{f}\right]$ with $T_{f}=T$ or $\left|y\left(T_{f}\right)-x_{0}\right|=M$. We estimate the distance between two such solutions on the interval where both solutions are defined, i.e. the shorter interval.

The following theorem is the main result of this section.

Theorem 3.1. The set $\mathcal{M}$ is residual in $C_{S D}(K)$, i.e. for almost all Scorza Dragoni right-hand sides (1.1) admits an unique (noncontinuable) solution.

The proof of Theorem 3.1 is given in the end of this section.

Corollary 3.2. Let $\left\{y_{i}(\cdot)\right\}_{i=1}^{\infty}$ be sequence of $\varepsilon_{i}$-solutions of (1.1) with right-hand side $f(\cdot, \cdot) \in \mathcal{M}_{M}$. If $\varepsilon_{i} \rightarrow 0^{+}$as $i \rightarrow \infty$, then $y_{i}(\cdot)$ converges uniformly to the unique solution $y(\cdot)$ of (1.1).

Notice that Corollary 3.2 implies continuous dependence of the solution on the right-hand side. Using the same method as in the proof of Theorem 3.1 one 
can prove that for every $f(\cdot, \cdot) \in \mathcal{M}$ if $\left\{x^{m}(\cdot)\right\}_{m=1}^{\infty}$ is a sequence of solutions of (1.1) with initial conditions $x_{m}$ respectively such that $\lim _{m \rightarrow \infty} x_{m}=x_{0}$, then $y^{m}(\cdot)$ converges uniformly to the unique solution $x(\cdot)$ of (1.1) with initial condition $x_{0}$.

The following result is a trivial consequence of Theorem 3.1.

Corollary 3.3. Let $K$ be separable. Then for almost every Caratheodory right-hand side the differential equation (1.1) has unique (noncontinuable) solution.

The proof follows from the fact that every Caratheodory function is Scorza Dragoni in case of separable $K$.

Definition 3.4. An absolutely continuous function $x(\cdot)$ is said to be $\varepsilon^{-}$ solution of (1.1) if it satisfies

$$
\dot{x}(t)=f(t, x(t))+l(t) \quad \text { a.e. on } I, \quad x(0)=x_{0},
$$

where $l(\cdot)$ is a strongly measurable function with $|l(t)| \leq 2 \sup _{x \in \mathbb{B}_{M}}|f(t, x)|$ such that $\int_{I}|l(t)| d t \leq \varepsilon$.

Proposition 3.5. System (1.1) admits $\varepsilon$-solution for every $\varepsilon>0$ and every $f \in C_{S D}\left(\mathbb{B}_{M}\right)$.

Proof. Let fix $\varepsilon>0$, and $f \in C_{S D}\left(\mathbb{B}_{M}\right)$. Then there exists a positive $L_{1}$ function $r(\cdot)$ such that $r(t) \geq 3 \sup _{x \in \mathbb{B}_{M}}|f(t, x)|$ for a.a. $t \in I$.

Since $r(t)>0$, then the function $g(t)=\int_{0}^{t} r(s) d s$ is strictly increasing. Thus the equation $g(t)=M$ admits unique solution $T_{M}$. Next set $T_{M}=T$ it $g(T)<M$. There exists $\delta$ such that $\int_{J} r(t) d t<\frac{\varepsilon}{25}$ for every $J \subset I$ with meas $(J)<\delta$. Let $I_{\delta} \subset\left[0, T_{M}\right]$ be open with meas $\left(I_{\delta}\right)<\delta$ such that $f(\cdot, \cdot)$ is continuous on $\left(I \backslash I_{\delta}\right) \times \mathbb{B}_{M}$.

Let the required $\varepsilon$-solution $y(\cdot)$ exists on $[0, b)$, where $b \in\left(0, T_{M}\right)$. Since $|\dot{y}(t)| \leq r(t)$, one has that the limit $y(b)=\lim _{t \rightarrow b-0} y(t)$ exists. (It is possible that $b=0$ and in this case the interval is not semiopen.)

Consider now the case when $y(\cdot)$ is defined on $[0, b]$ with $b \in\left[0, T_{M}\right)$ and $\left|y(b)-x_{0}\right|<M$. There are two opportunities:

1) $b \in I_{\delta}$, i.e. $b \in\left(a_{k}, b_{k}\right)$, where $I_{\varepsilon}=\bigcup_{k=1}^{\infty}\left(a_{k}, b_{k}\right)$; define $y(t)=y(b)$. 
2) $b \in\left(I \backslash I_{\delta}\right)$; define $y(t)=y(b)+\int_{b}^{t} \nu(s) d s$, where

$$
\nu(s)= \begin{cases}f(b, y(b)) & s \in\left[b, T_{M}\right] \bigcap\left(I \backslash I_{\delta}\right) \\ f(s, y(b)) & s \in\left[b, T_{M}\right] \bigcap I_{\delta} .\end{cases}
$$

Since $f(\cdot, \cdot)$ is continuous on $\left(I \backslash I_{\delta}\right) \times \mathbb{B}_{M}$, there exists $\tau>0$ such that $|f(b, y(b))-f(t, y(t))| \leq \varepsilon$ on $[b, b+\tau]$. Consequently $y(\cdot)$ is $\varepsilon$-solution on $[0, b+\tau]$.

One can extend $y(\cdot)$ up to the boundary of $I \times \mathbb{B}_{M}$ by simple application of Zorn's lemma. If $y(\cdot)$ is defined on $\left[0, T_{f}\right]$, where $T_{f}<T$ and $\left|y\left(T_{f}\right)-y(0)\right|<M$, then one can consider the equation (1.1) on $\left[T_{f}, T\right]$ and extend $y(\cdot)$ on an interval larger than $\left[0, T_{f}\right]$. Applying Zorn's lemma one can prove that $y(\cdot)$ exists on an interval $\left[0, T_{y}\right]$ such that $T_{y}=T$ or $\left|y\left(T_{y}\right)-x_{0}\right|=M$.

The following theorem is proved in [10].

Theorem 3.6. If $f(\cdot, \cdot)$ is locally Lipschitz, then (1.1) has unique solution $x(\cdot)$ and if $\left\{x_{\varepsilon}(\cdot)\right\}_{\varepsilon>0}$ is a net of $\varepsilon$-solutions then $x_{\varepsilon}(t) \rightarrow x(t)$ as $\varepsilon \rightarrow 0$, uniformly on some interval $[0, a]$.

Now we are ready to prove the main result using Proposition 3.5 and Theorem 3.6.

Proof. (of Theorem 3.1.) First, we will prove that $\mathcal{M}_{M}$ is residual in $C_{S D}\left(\mathbb{B}_{M}\right)$.

Let $h \in C_{S D}\left(\mathbb{B}_{M}\right)$. Denote by $\operatorname{Sol}(\varepsilon, h)$, the set of all $\varepsilon$-solutions of (1.1) with right-hand side $h$. Here note that different $\varepsilon$-solutions can be defined on different intervals. Denote by $\left[0, T_{h}^{\varepsilon}\right]$ the maximal interval where all elements of $\operatorname{Sol}(\varepsilon, h)$ are defined and the distance between two different $\varepsilon$-solutions is the supremum norm of their difference on $\left[0, T_{h}^{\varepsilon}\right]$.

Denote by $S S(m)$ the set of all $h(\cdot, \cdot) \in C_{S D}\left(\mathbb{B}_{M}\right)$ such that $\operatorname{diam}(\operatorname{Sol}(\varepsilon, h)) \geq$ $\frac{1}{m}$, for every $\varepsilon>0$, where $m$ is a natural number. Clearly, the differential equation (1.1) admits an unique solution if and only if $h \notin S S(m)$ for every natural $m$.

Suppose $g(\cdot, \cdot) \in C_{S D}\left(\mathbb{B}_{M}\right)$. Let $y(\cdot)$ be an $\varepsilon$-solution of

$$
\dot{y}(t)=g(t, y(t)), y(0)=x_{0} .
$$

If $\rho_{M}(h, g)<\delta$, then $y(\cdot)$ is $\varepsilon+\delta$-solution of (1.1) with right-hand side $h$, because:

$$
\int_{I}|\dot{y}(t)-h(t, y(t))| d t \leq \int_{I}|\dot{y}(t)-g(t, y(t))| d t+\rho_{M}(h, g) .
$$


Let $\left\{g_{k}(\cdot, \cdot)\right\}_{k=1}^{\infty} \subset S S(m)$, then $\operatorname{diam}\left(\operatorname{Sol}\left(\varepsilon, g_{k}\right)\right) \geq \frac{1}{m}$ for every $\varepsilon>0$. Let $g_{k}(\cdot, \cdot) \rightarrow g(\cdot, \cdot)$ w.r.t. $\rho_{M}$. If $\rho_{M}\left(g_{k}, g\right)<\delta$, then $\left.\operatorname{Sol}\left(\varepsilon, g_{k}\right)\right) \subset \operatorname{Sol}(\varepsilon+\delta, g)$. However, $\delta>0$ is arbitrary and $\operatorname{diam}\left(\operatorname{Sol}\left(\varepsilon, g_{k}\right)\right) \geq \frac{1}{m}$, hence $\operatorname{diam}(\operatorname{Sol}(\varepsilon, g)) \geq$ $\frac{1}{m}$, i.e. $g \in S S(m)$. So, $S S(m)$ is closed w.r.t. $\rho_{M}$. Furthermore, if $f(\cdot, \cdot)$ is locally Lipschitz on $I \times \mathbb{B}_{M}$, then $f \notin S S(m)$. Therefore, $S S(m)$ has empty interior, thanks to Corollary 2.4 .

It is standard to show that $\bigcap_{M=1}^{\infty} \mathcal{M}_{M}=\mathcal{M}$, thus the proof is complete.

\section{Applications}

In this section we apply our result to set differential equations, differential inclusions and also to fuzzy differential equations.

\subsection{Set Differential Equations}

The set differential equations have been studied since more than 40 years (see [2]). We refer the reader to [8], where these equations are comprehensively studied. The recent interesting (unfortunately in Russian) book [12] studies set valued and fuzzy differential equations.

Further they have been applied to theory of differential inclusions (cf. [15]) and fuzzy differential equations [12].

Let $E$ be Banach space. The Hausdorff distance between closed bounded subsets of $E$ is

$$
D_{H}(A, B)=\max \left\{\sup _{a \in A} \inf _{b \in B}|a-b|, \sup _{b \in B} \inf _{a \in A}|a-b|\right\} .
$$

The continuity concepts will be understood w.r.t. Hausdorff metric.

Denote by $C C(E)$ the set of all convex compact subsets of $E$. Given a function $F: I \rightarrow C C(E), F$ is said to be differentiable in the sense of Hukuhara at the point $t^{\prime}$ if there exists $A \in C C(E)$ such that

$$
A=\lim _{h \rightarrow 0^{+}} \frac{F\left(t^{\prime}+h\right) \ominus F\left(t^{\prime}\right)}{h}=\lim _{h \rightarrow 0^{+}} \frac{F\left(t^{\prime}\right) \ominus F\left(t^{\prime}-h\right)}{h},
$$

where $B \ominus C=D$ implies $C+D=B$. The Hukuhara derivative is denoted by $D^{h}$. 
Consider the following set differential equation:

$$
D^{h} Y(t)=G(t, Y(t)), Y(0)=Y_{0},
$$

where $G: I \times C C(E) \rightarrow C C(E)$. It is well known (cf. [14]) that the set of all convex compact subsets of $E$ can be embedded as a closed convex cone in a (nonseparable) Banach space $X$. The metric $\varrho$ on $C_{S D}(C C(E))$ is defined as in (1.2), where $\varrho_{M}$ is given by

$$
\varrho_{M}(F, G)=\int_{I} \sup _{|x| \leq M} D_{H}(F(t, x), G(t, x)) d x .
$$

By Theorem 3.1 we get the following

Corollary 4.1. The set of all Scorza Dragoni multifunctions $G$ for which (4.1) admits unique solution is residual in $C_{S D}(C C(E))$.

Now we will study the following differential inclusion:

$$
\dot{x}(t) \in F(t, x(t)), x(0)=x_{0}, t \in I .
$$

Here $F: I \times E \rightarrow C C(E)$ is a Scorza Dragoni multifunction.

As usual, a solution of (4.2) is an absolute continuous function $x(\cdot)$ which satisfies (4.2) for a.a. $t \in I$. We associate to (4.2) the following set differential equation

$$
D^{h} Y(t)=G(t, Y(t)), Y(0)=x_{0},
$$

where $G: I \times C C(E) \rightarrow C C(E)$ is defined as $G(t, A)=\overline{c o} F(t, A)$. It is shown in [15] that $G$ is Scorza Dragoni if $F$ is. Let $\lim _{n \rightarrow \infty} \varrho\left(F_{n}, F\right)=0$. Then it is easy to see that $D_{H}\left(G_{n}(t, A), G(t, A)\right) \leq \sup _{x \in \mathbb{B}_{M}} D_{H}\left(F_{n}(t, x), F(t, x)\right)$ for every $A \subset B_{M}$. Consequently $\varrho_{M}\left(G_{n}, G\right) \rightarrow 0$ as $n \rightarrow \infty$.

Using the same method (with obvious modifications) as in previous section one can show that the set of all Scorza Dragoni multifunctions $F$ for which the set differential equation (4.3) admits unique solution is residual. From Theorem 2.8.1 of [15] we know that the differential inclusion

$$
\dot{x}(t) \in \overline{e x t} F(t, x), x(0)=x_{0}
$$

admits a solution if (4.3) has a solution. Here ext $A$ denotes the set of all extreme points of $A$. Therefore the following theorem is true:

Theorem 4.2. The set of all (integrally bounded) Scorza Dragoni maps $F(\cdot, \cdot)$ with convex compact values such that the differential inclusion (4.4) has a (global) solution, is residual. 
Remark 4.3. It is proved in [5] that in case $E \equiv \mathbb{R}^{n}$ for almost all Scorca Dragoni $F(\cdot, \cdot)$ with convex compact values the solution set of (4.4) is dense in the solutions set of:

$$
\dot{x}(t) \in F(t, x(t)), x(0)=x_{0} .
$$

A very interesting and open question is the extension ot that result in case of infinite dimensional spaces.

\subsection{Application to Fuzzy Differential Equations}

Theory of fuzzy differential equations rapidly grows. We recall only the main concepts and refer the reader to [9] or [12].

Denote the space of fuzzy numbers by $\mathbb{E}=\left\{x: \mathbb{R}^{n} \rightarrow[0,1] ; x\right.$ satisfies 1$\left.\left.)-4\right)\right\}$ :

1) $x$ is normal, i.e., there exists $y_{0} \in \mathbb{R}^{n}$ such that $x\left(y_{0}\right)=1$,

2) $x$ is fuzzy convex i.e. $x(\lambda y+(1-\lambda) z) \geq \min \{x(y), x(z)\}$ whenever $y, z \in \mathbb{R}^{n}$ and $\lambda \in[0,1]$,

3) $x$ is upper semicontinuous i.e. for any $y_{0} \in \mathbb{R}^{n}$ and $\varepsilon>0$ there exists $\delta\left(y_{0}, \varepsilon\right)>0$ such that $x(y)<x\left(y_{0}\right)+\varepsilon$ whenever $\left|y-y_{0}\right|<\delta, y \in \mathbb{R}^{n}$,

4) The closure of the set $\left\{y \in \mathbb{R}^{n} ; x(y)>0\right\}$ is compact.

The set $[x]^{\alpha}=\left\{y \in \mathbb{R}^{n} ; x(y) \geq \alpha\right\}$ is called $\alpha$-level set of $x$.

It follows from 1) - 4) that the $\alpha$-level sets $[x]^{\alpha}$ are in $C C\left(\mathbb{R}^{n}\right)$ for all $\alpha \in(0,1]$, where $C C\left(\mathbb{R}^{n}\right)$ denotes the compact convex subsets of $\mathbb{R}^{n}$. The fuzzy zero is $\hat{0}(y)=\left\{\begin{array}{l}0 \text { if } y \neq 0, \\ 1 \text { if } y=0 .\end{array}\right.$

The metric in $\mathbb{E}$ is defined by $D(x, y)=\sup _{\alpha \in(0,1]} D_{H}\left([x]^{\alpha},[y]^{\alpha}\right)$, where $D_{H}(\cdot, \cdot)$ means the Hausdorff distance in $C C\left(\mathbb{R}^{n}\right)$.

The map $x: I \rightarrow \mathbb{E}$ is said to be differentiable at $\hat{t} \in I$ if there exists a fuzzy number $u$ such that

$$
\lim _{t \rightarrow 0^{+}} \max \{D(x(\hat{t}+h), x(\hat{t})+h u), D(x(\hat{t}), x(\hat{t}-h)+h u)\}=0
$$

(compare to [13]). The fuzzy differential equations are comprehensively studied in [9] (see also [12]).

The set $\mathbb{E}$ is a complete semilinear metric space with respect to metric $D(\cdot, \cdot)$. This space is not locally compact and nonseparable. We know from Theorem 2.1 in [7] that $\mathbb{E}$ can be embedded as a closed convex cone in a Banach space $\mathfrak{X}$. The embedding map $j: \mathbb{E} \rightarrow \mathfrak{X}$ is isometry and isomorphism and hence $f: I \rightarrow \mathbb{E}$ is continuous iff $j(f)(\cdot)$ is continuous. Furthermore, $j(\cdot)$ preserves 
differentiation and integration. Namely if $\dot{f}(t)$ exists then $\frac{d}{d t} j(f)(t)$ also exists and $j(\dot{f})(t)=\frac{d}{d t} j(f)(t)$, where $\frac{d}{d t}$ is the usual differential operator.

Now if $g(\cdot): I \rightarrow \mathbb{E}$ is strongly measurable and integrable then $j(g)(\cdot)$ is strongly measurable and Bochner integrable and

$$
j\left(\int_{0}^{t} g(s) d s\right)=\int_{0}^{t} j(g)(s) d s \text { for all } t \in I .
$$

Consider the fuzzy differential equation:

$$
\dot{z}(t)=g(t, z(t)), z(0)=z_{0}, t \in[0, T] .
$$

Associate to (4.7) the ordinary differential equation

$$
\dot{x}(t)=f(t, x(t)), x(0)=x_{0},
$$

where $x(t)=j(z(t))$ and $f(t, x)=g(t, j(z))$.

The following result is a corollary of Theorem 3.1.

Theorem 4.4. The fuzzy differential equation (4.7) has an unique solution for almost all (in Baire sense) Scorza Dragoni function $g(\cdot, \cdot)$. This solution depends continuously on $g$ and $z_{0}$.

Remark 4.5. Due to some bad properties of Hukuhara derivative, new concept of generalized derivative of fuzzy maps is studied (see [1]). In this case $x(\cdot)$ is said to be differentiable at $\hat{t}$ with derivative $u \in \mathbb{E}$ if $(4.5)$ or

$$
\lim _{t \rightarrow 0^{+}} \max \{D(x(\hat{t}-h), x(\hat{t})+(-h) h u), D(x(\hat{t}), x(\hat{t}+h)+(-h) u)\}=0
$$

holds true, that is, however out of the scope of the paper. Notice only that it is impossible to prove stronger existence result with the help of generalized derivative.

\section{Acknowledgments}

The present research was supported by a grant to the project BG051P00013.3.06 under sponsorship of EU. 


\section{References}

[1] Bede B., Gal S.G., Generalizations of the differentiability of fuzzy-numbervalued functions with applications to fuzzy differential equations, Fuzzy Sets and Systems 151 (2005) 581-599.

[2] De Blasi F., Iervolino F., Equazioni differential con soluzioni valore compacto convesso, Boll. Unione Mat. Ital 2 (1969) 491-591.

[3] De Blasi F., Myjak J., The generic property of existence of solutions for a class of multivalued differential equations in Hilbert spaces, Funkcial. Ekvac. 21 (1978) 271-278.

[4] De Blasi F., Myjak J., Orlicz type category results for differential equations in Banach spaces, Comment. Math. Prace Mat. 23 (1983) 193-197.

[5] T. Donchev, Generic properties of multifunctions: application to differential inclusions, Nonlinear Anal. 74 (2011) 2585-2590.

[6] Dugundji, I., An extension of Tietze's theorem, Pacific J. Math. 1 (1951) 353-367.

[7] Kaleva O., Fuzzy differential equations, Fuzzy Sets and Systems 35 (1990) 389-396.

[8] Lakshmikantham V., Gnana Baskar T., Vasundhara Devi J., Theory of Set Differential Equations in Metric Spaces, Cambridge Scientific Publishers, 2006.

[9] Lakshmikantham V., Monhapatra R., Theory of Fuzzy Differential Equations and Inclusions, Taylor \& Francis, London, 2003.

[10] Lasota A., Yorke J.A., The generic properties of existence of solutions of differential equations in Banach space, J. Differential Equations 13 (1973) 1-12.

[11] Mallet-Paret J., Generic properties of retarded functional differential equations, Bull. Amer. Math. Soc. 81 (1975) 750-752.

[12] Plotnikov A., Skripnik N., Differential Equations with Clear and Fuzzy Multivalued right-hand Side, Astroprint, Odessa, 2009 (in Russian).

[13] Puri M., Ralescu D., Differentiation of fuzzy functions, J. Math. Anal. Appl. 91 (1986) 400-422. 
[14] Radström H., An embedding for space of convex sets, Proc. Amer. Math. Soc. 3 (1952) 165-169.

[15] Tolstonogov A., Differential Inclusions in Banach Space, Kluwer, Dordrecht, 2000.

[16] Yu J., Yuan G. X.-Z., Isac G., The stability of solutions for differential inclusions and differential equations in the sense of Baire category theory, Appl. Math. Lett. 11 (1998) 51-56. 
